\title{
Propofol sedation for flexible bronchoscopy: a randomised, noninferiority trial
}

\author{
Peter Grendelmeier, Michael Tamm, Eric Pflimlin and Daiana Stolz
}

\section{Affiliation:}

Clinic of Pulmonary Medicine and Respiratory Cell Research, University Hospital Basel, Basel, Switzerland.

\section{Correspondence:}

D. Stolz, Clinic of Pulmonary Medicine and Respiratory Cell Research, University Hospital Basel, Petersgraben

4, 4031 Basel, Switzerland.

E-mail: daiana.stolzQusb.ch

ABSTRACT Propofol has been established as a reliable method for sedation in flexible bronchoscopy. There are no data comparing propofol administered as intravenous boluses versus continuous infusion.

702 consecutive patients undergoing flexible bronchoscopy were randomly allocated to receive intravenous propofol using either an intermittent bolus technique or a continuous infusion. The primary end-point was the number of adverse events assessed at the end of flexible bronchoscopy and at $24 \mathrm{~h}$.

The number of any adverse event was similar in both randomised groups (219 versus $211, \mathrm{p}=0.810$ ). There were complications in eight cases (seven major bleedings, one respiratory failure). As compared with the bolus group, the amount of propofol required was significantly higher in the infusion group (226 $\pm 147 \mathrm{mg}$ versus $308 \pm 204.8 \mathrm{mg}, \mathrm{p}<0.0001)$. In a multivariate regression model, this difference remained significant independent of the duration and the interventions performed during the procedure. The duration of bronchoscopy was significantly longer in the infusion group (median 14 (interquartile range 9-24) versus $17(12-27)$ min, $\mathrm{p}<0.0001)$.

Propofol continuous infusion is as safe as bolus administration; however, it is associated with higher propofol requirements and a longer duration of the bronchoscopy.

@ERSpublications

Propofol continuous infusion is as safe as bolus administration, but has higher requirements and longer bronchoscopy http://ow.ly/r4Uas

Received: Dec 122012 | Accepted after revision: July 022013 | First published online: July 302013

Clinical trial: This study is registered at www.controlled-trials.com with identifier number ISRCTN66129676.

Support statement: D. Stolz was supported by grants from the Swiss National Foundation (PP00P3_128412/1). Additional funding was provided by the Clinic of Pulmonary Medicine and Respiratory Cell Research, University Hospital Basel, Basel, Switzerland.

Conflict of interest: None declared. 


\section{Introduction}

The British Thoracic Society states that sedation for flexible bronchoscopy should be offered to patients where there is no contraindication [1]. The aim of sedation is to facilitate patient comfort and satisfaction and to alleviate patient anxiety, cough and dyspnoea while reducing complications of the procedure [2-4]. According to a survey of registered members of the British Thoracic Society, $>95 \%$ of centres routinely perform sedated bronchoscopy [5]. Optimal sedation for flexible bronchoscopy has been assessed in a number of studies evaluating different sedative drug regimens using single agents or combinations thereof [6-10]. Propofol (2,6-di-isopropylphenol), a sedative hypnotic, has recently proved to be a safe and attractive alternative to combined sedation with midazolam and hydrocodone due to its rapid onset of action and fast recovery time, particularly if timely discharge was a priority [11-18]. Additionally, as compared with midazolam alone, propofol seems to provide a higher quality of sedation in terms of neuropsychometric recovery and patient tolerance [18]. However, unlike the benzodiazepines, propofol does not have a reversal agent. Many specialty bodies recommend its use only by those trained in the administration of anaesthesia, and the license of whom propofol may be administered by also differs by licensing agency.

The administration of propofol for conscious sedation in flexible bronchoscopy is usually performed by repeated intravenous boluses. In contrast, the continuous infusion of propofol is an established method of sedation in the intensive care unit (ICU), where its administration occurs over hours or days. Taking into account the increasing complexity and, thus, duration of diagnostic and interventional flexible bronchoscopy, continuous infusion of propofol seems to be an appealing approach to conscious sedation in this setting.

As yet, there are very limited data of propofol in gastrointestinal endoscopy $[19,20]$ and no data comparing bolus administration versus continuous infusion of propofol in flexible bronchoscopy. Therefore, a large, prospective, randomised, noninferiority trial was undertaken in order to determine whether propofol given as a continuous infusion is as effective and safe as propofol applied by bolus administration in patients undergoing flexible bronchoscopy.

\section{Methods}

A total of 1223 consecutive patients were assessed for eligibility. 43\% of screened patients did not meet eligibility criteria for study inclusion (fig. 1). The main reasons for noninclusion were bronchoscopy at the ICU and emergency bronchoscopy. Thus, 702 patients undergoing flexible bronchoscopy were randomly allocated to receive intravenous propofol using either a continuous infusion or an intermittent bolus technique. Patients aged $\geqslant 18$ years were included between April 2011 and January 2012. Intubated or isolated patients, patients with known allergy or intolerance to propofol, patients undergoing emergency bronchoscopy, pregnant or breastfeeding females and patients with a mental disorder preventing appropriate judgment concerning study participation were not included in the study. Informed consent was obtained from each patient and the study was approved by the institutional review board, Ethikkommission beider Basel. The trial was registered with the Current Controlled Trials Database (ISRCTN66129676).

All patients were assessed by a member of the nursing team trained in anaesthesiology and a chest physician prior to the procedure, which included gradation of physical status in accordance with the American Society of Anesthesiologists (ASA) criteria. Current medication such as anticoagulants, antiplatelet drugs, sedatives and hypnotics were recorded. Comorbidities including chronic obstructive pulmonary disease, coronary artery disease, congestive heart failure, cerebrovascular disease, renal failure, liver disease, malignant solid tumour, haematological malignancy, rheumatic disease, diabetes mellitus, alcohol abuse and HIV infection were noted and current blood work results were listed.

Bronchoscopy procedures were performed transnasally or transorally, with the patients in the semi-recumbent position, by a total of five pulmonary fellows under close supervision of five pulmonary attending physicians. Electrocardiographic and transcutaneous pulse oxymetric monitoring were recorded continuously during the procedure. In addition, automated noninvasive blood pressure measurements were performed every 5 min. Supplemental oxygen was given at $4 \mathrm{~L} \cdot \mathrm{min}^{-1}$ via a nasal cannula to all patients. In the case of desaturation to $\leqslant 90 \%$, oxygen delivery was increased to $6 \mathrm{~L} \cdot \mathrm{min}^{-1}[21]$. Patients were routinely given $4 \mathrm{mg} i . v$. hydrocodone immediately prior to flexible bronchoscopy, as previously described [10]. Nasal anaesthesia was achieved by $2 \%$ lidocaine gel. Bronchoscopists were advised to instil 3-mL aliquots of $1 \%$ lidocaine over the vocal cords and on the trachea and both right and left main bronchi. Instilled lidocaine doses were recorded for each patient. All doses of supplemental local anaesthesia required, as judged by the bronchoscopist, were recorded for each patient. No inhaled lidocaine was given prior to the procedure [7].

Patients were randomly assigned to either intravenous propofol using an intermittent bolus technique or as continuous infusion for conscious sedation. Randomisation was through arbitrary allocation to one of the two treatment groups based on a computer-generated random list (GraphPad Prism; GraphPad Software, 


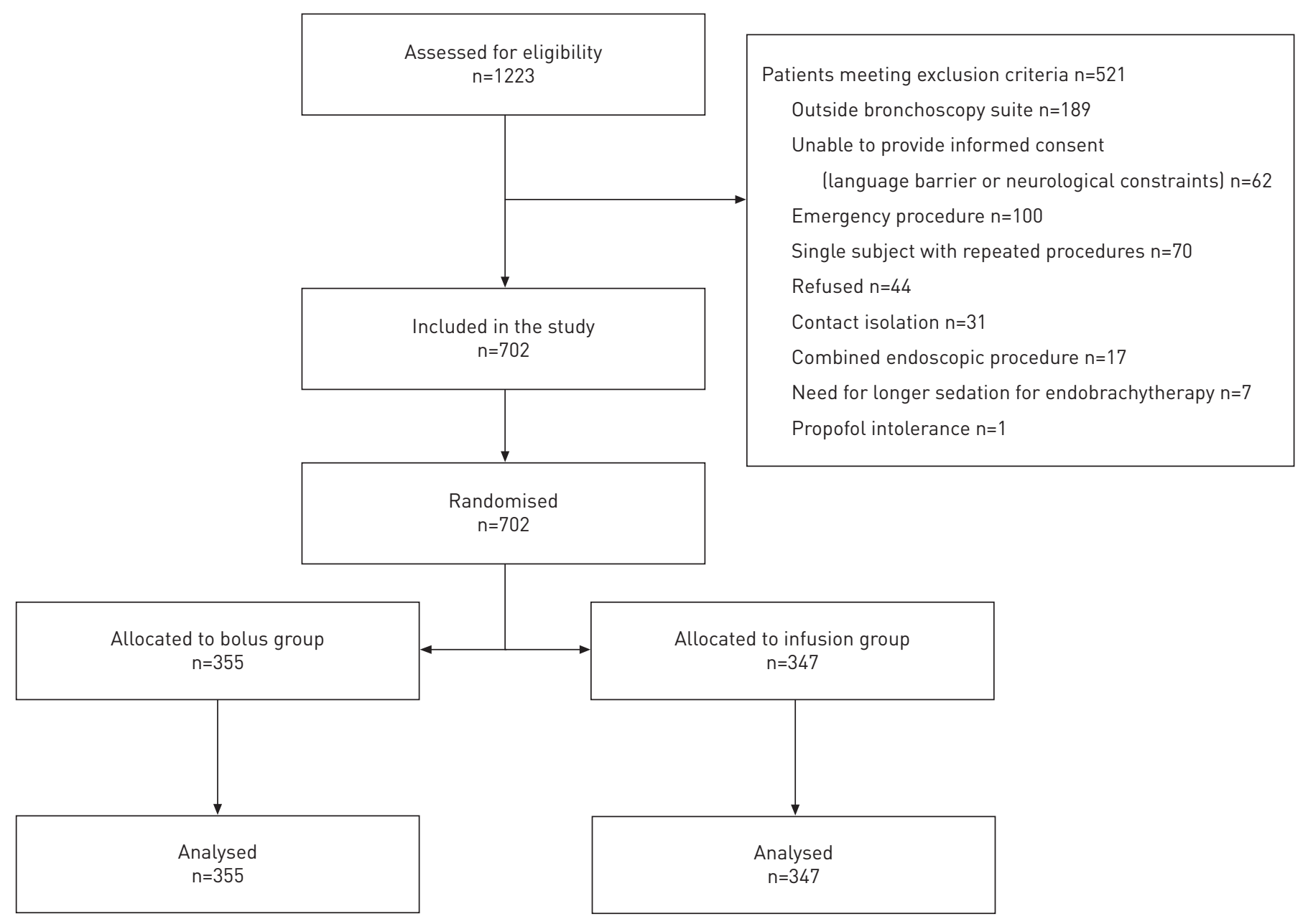

FIGURE 1 Study flow chart for patients included in the study.

Inc., San Diego, CA, USA). Every patient's assignment was carried out in the waiting room of the bronchoscopy suite by a research nurse.

For patients assigned to the bolus administration of propofol (bolus group), the loading doses of propofol were titrated in order to achieve adequate conscious sedation (onset of ptosis for bronchoscopy). Patients received an initial $20 \mathrm{mg}$ of $i . v$. propofol, followed by a carefully titrated dose. For ASA I and II patients, the steps comprised 10-20 mg i.v. propofol, whereas for ASA III and IV, exactly $10 \mathrm{mg}$ i.v. propofol was administered based on the clinical response, as previously described [22]. Between each bolus, a pause lasting $\geqslant 20 \mathrm{~s}$ had to be observed. Additional i.v. boluses of propofol were given, if the effect disappeared during the examination, depending on the clinical effect, in order to maintain the required level of sedation. Signs of pain or discomfort, agitation, persistent cough, and inadequate motor or verbal response to manipulation were considered indicators of insufficient sedation, leading to administration of an additional dose of propofol (10-20 mg). The total dose of propofol was documented for each patient.

Patients assigned to the continuous infusion of propofol (infusion group) received an initial bolus of $10 \mathrm{mg}$ i.v. propofol, immediately followed by the continuous infusion of propofol at an initial rate of $0.3 \mathrm{mg} \cdot \mathrm{kg}^{-1} \cdot \mathrm{min}^{-1}$. The infusion rate was reduced to $0.2 \mathrm{mg} \cdot \mathrm{kg}^{-1} \cdot \mathrm{min}^{-1}$ after $3 \mathrm{~min}$ and was further diminished to $0.1 \mathrm{mg} \cdot \mathrm{kg}^{-1} \cdot \mathrm{min}^{-1}$ and to $0.05 \mathrm{mg} \cdot \mathrm{kg}^{-1} \cdot \mathrm{min}^{-1}$ after another 3 and $6 \mathrm{~min}$, respectively, if conscious sedation was achieved. In case of inadequate sedation, a bolus of 10-20 mg of propofol was given and the infusion rate was increased in reversed order to a maximum rate of $0.5 \mathrm{mg} \cdot \mathrm{kg}^{-1} \cdot \mathrm{min}^{-1}$. In case of apnoea, hypoxaemia or hypotension, the continuous infusion could be reduced in the above mentioned manner or completely stopped at all times as judged by the bronchoscopist. Applied propofol infusion rates were based on the analysis of previous data in a different patient population [17]. Propofol was administered by the bronchoscopy nurse at the endoscopist's discretion. The use of propofol in the department was introduced almost 10 years ago under the supervision of a board-certified anaesthetist who 
was personally present during a 3-month initiation period. Since the introduction of this sedation method, propofol has been administered by a bronchoscopy nurse with training in deep sedation. Currently all endoscopy nurses are specially trained in the administration of propofol and the risks associated with its use. They are proctored while administering propofol by an experienced nurse, until proficiency with administration is demonstrated. All nurses are carefully instructed in the management of emergency situations (e.g. mask ventilation, positioning of a nasopharyngeal tube). In every endoscopy procedure room, equipment for emergency mask ventilation and appropriate drugs for use during emergency situations were always immediately to hand.

Diagnostic procedures, i.e. brushing, washings, bronchoalveolar lavage (BAL), mediastinal as well as peripheral transbronchial needle aspiration, endobronchial and transbronchial biopsy and endobronchial ultrasound, were performed dependent upon the clinical indication. Additionally, interventions such as stent or endobronchial valves implantation and laser therapy were carried out. Haemodynamic parameters, sedation requirements, duration of bronchoscopy, bronchoscopic procedures and complications were noted during the procedure on a form specifically designed for the study.

Adverse events were defined as oxygen desaturation $\leqslant 90 \%$, need for nasopharyngeal or oropharyngeal airway insertion, hypotension with a systolic blood pressure of $<90 \mathrm{mmHg}$, pneumothorax and minor bleeding. Complications were defined as major bleeding, need to abort bronchoscopy, need for intubation, need for ICU transfer post-bronchoscopy and death.

At the end of the procedure, bronchoscopists and nursing staff would chart their perception of cough during the procedure on a $10-\mathrm{cm}$ visual analogue scale (VAS). Similarly, $2 \mathrm{~h}$ after bronchoscopy, patients were also asked to record their perception of cough related to the procedure on a 10-cm VAS. On this scale, 0 denoted no cough and 10 represented incessant cough. Patients were also asked to record anxiety and discomfort associated with the procedure on a 10-cm VAS. On this scale, 0 denoted no fear or discomfort and 10 represented the greatest imaginable fear or discomfort. Willingness to undergo repeat flexible bronchoscopy was also documented $2 \mathrm{~h}$ after bronchoscopy.

Haemodynamic monitoring was performed immediately before, during and shortly after the procedure (after removal of the bronchoscope), as well as before transfer from the bronchoscopy suite to the recovery room. Moreover, the patient's blood pressure, cardiac frequency and respiratory rate were monitored for up to $3 \mathrm{~h}$ after bronchoscopy, until discharge.

The primary end-point was the number (percentage) of adverse events and complications (oxygen desaturation $\leqslant 90 \%$, need for nasopharyngeal or oropharyngeal airway insertion, need for intubation, hypotension with a systolic blood pressure of $<90 \mathrm{mmHg}$, minor or major bleeding, ICU need postbronchoscopy, pneumothorax, need to abort bronchoscopy and death) assessed by the study physician during and up to $24 \mathrm{~h}$ after the procedure.

Secondary pre-defined end-points included total dose of propofol, dose of propofol per kg body weight, dose of propofol per kg body weight and per minute, total dose of hydrocodone, total amount of lidocaine doses, duration of the procedure, mean lowest oxygen saturation during the procedure, mean lowest systolic blood pressure during the procedure, haemodynamic parameters other than blood pressure during and after the procedure, cough scores, as assessed by a VAS by patients, nurses and physicians $2 \mathrm{~h}$ after the procedure, patient discomfort, median patient overall well-being (comfort) at $2 \mathrm{~h}$ after the procedure, willingness to undergo a repeated procedure, assessed by a VAS $2 \mathrm{~h}$ after the procedure, and fear of undergoing a repeated procedure, assessed by a VAS $2 \mathrm{~h}$ after the procedure.

\section{Data analyses}

Assuming an incidence of complications of 0.36 [11] in the arm treated with propofol as bolus and incidence of complications of 0.31 in the arm treated with propofol in a continuous fashion, a total of 688 patients, 344 in each treatment arm, were considered to be needed to achieve a significance level of $<0.05$ with a power of 0.8 . Considering a $1 \%$ loss to follow-up, a total of 702 patients were aimed for inclusion.

Differences in dichotomous variables were evaluated using the Chi-squared test or Fischer's Exact test, as appropriate. Normally distributed parameters were analysed using the Student's t-test for equality of means. All other continuously non-normally distributed parameters were evaluated using the nonparametric Mann-Whitney U-test or Kruskal-Wallis test, as appropriate.

The SSPS version 19 (SSPS Inc., Chicago, IL, USA) program was used. All tests were two-tailed; a p-value of $<0.05$ was considered significant. Results are expressed as mean \pm SD or median (interquartile range) unless otherwise stated. 
TABLE 1 Demographic data of consecutive patients undergoing flexible bronchoscopy

\begin{tabular}{|c|c|c|c|c|}
\hline Characteristics & Bolus & Infusion & Total & p-value \\
\hline Subjects $\mathrm{n}$ & 355 & 347 & 702 & \\
\hline Age years & $61.2 \pm 15$ & $61.9 \pm 14$ & $61.5 \pm 15$ & 0.556 \\
\hline Males & $207(58.3)$ & $197(56.8)$ & $404(57.5)$ & 0.680 \\
\hline Height $\mathrm{cm}$ & $170 \pm 10.2$ & $170 \pm 10.4$ & $170 \pm 10.3$ & 0.762 \\
\hline Weight kg & $71.5 \pm 17.5$ & $70.3 \pm 17.7$ & $70.8 \pm 17.6$ & 0.350 \\
\hline $\mathrm{BMI} \mathbf{k g} \cdot \mathrm{m}^{-2}$ & $24.7 \pm 5.3$ & $24.3 \pm 5.5$ & $24.5 \pm 5.4$ & 0.340 \\
\hline \multicolumn{5}{|l|}{ Smoking status \% } \\
\hline Never-smoker & $104(29.7)$ & $106(30.9)$ & $210(30.3)$ & \\
\hline Current smoker & $79(22.6)$ & $70(20.4)$ & $149(21.5)$ & 0.782 \\
\hline Ex-smoker & $167(47.7)$ & $167(48.7)$ & $334(48.2)$ & \\
\hline Pack-years n & $29 \pm 30.5$ & $27 \pm 30$ & $28 \pm 30.1$ & 0.587 \\
\hline \multicolumn{5}{|l|}{ ASA class $\%$} \\
\hline I & $7(2.0)$ & $4(1.2)$ & $11(1.6)$ & \\
\hline ॥ & $66(18.6)$ & $77(22.2)$ & $143(20.4)$ & 0.514 \\
\hline III & $255(71.8)$ & $241(69.5)$ & $496(70.7)$ & \\
\hline IV or V & $19(5.4)$ & $13(3.7)$ & $32(4.6)$ & \\
\hline \multicolumn{5}{|l|}{ Comorbidities \% } \\
\hline COPD & $119(33.5)$ & $117(33.7)$ & $236(33.6)$ & 0.909 \\
\hline Coronary artery disease & $52(14.6)$ & $45(13.0)$ & $97(13.8)$ & 0.519 \\
\hline Congestive heart failure & $25(7.0)$ & $18(5.2)$ & $43(6.1)$ & 0.310 \\
\hline Cerebral vascular disease & $12(3.4)$ & $9(2.6)$ & $21(3.0)$ & 0.541 \\
\hline Diabetes mellitus & $47(13.2)$ & $37(10.7)$ & $84(12.0)$ & 0.280 \\
\hline Renal failure & $45(12.7)$ & $42(12.1)$ & $87(12.4)$ & 0.807 \\
\hline Liver disease & $8(2.3)$ & $10(2.9)$ & $18(2.6)$ & 0.598 \\
\hline Solid malignant tumour & $142(40.0)$ & $149(43.0)$ & $291(41.5)$ & 0.429 \\
\hline Haematological malignancy & $57(16.1)$ & $40(11.5)$ & $97(13.8)$ & 0.082 \\
\hline Immunosuppression & $111(31.3)$ & $89(25.6)$ & $200(28.5)$ & 0.099 \\
\hline Rheumatological disease & $22(6.2)$ & $17(4.9)$ & $39(5.6)$ & 0.447 \\
\hline HIV & $9(2.5)$ & $6(1.7)$ & $15(2.4)$ & 0.457 \\
\hline Alcohol abuse & $27(7.6)$ & $22(6.3)$ & $49(7.0)$ & 0.511 \\
\hline Intravenous drug use & $5(1.4)$ & $6(1.7)$ & $11(1.6)$ & 0.736 \\
\hline \multicolumn{5}{|l|}{ Current medication \% } \\
\hline Acetylsalicylic acid & $68(19.2)$ & $56(16.2)$ & $124(17.7)$ & 0.311 \\
\hline Clopidogrel & 8 (2.3) & $11(3.2)$ & $19(2.7)$ & 0.454 \\
\hline Prasugrel & $0(0)$ & $2(0.6)$ & $2(0.3)$ & 0.145 \\
\hline Oral anticoagulant & $32(9.0)$ & $20(5.8)$ & $52(7.4)$ & 0.100 \\
\hline Heparin (therapeutic dose) & $5(1.4)$ & $2(0.6)$ & $7(1.0)$ & 0.267 \\
\hline Heparin (prophylactic dose) & $15(4.2)$ & $9(2.6)$ & $24(3.4)$ & 0.234 \\
\hline LMWH (therapeutic dose) & $10(2.8)$ & $5(1.4)$ & $15(2.1)$ & 0.207 \\
\hline LMWH (prophylactic dose) & $77(21.8)$ & $66(19.0)$ & $143(20.4)$ & 0.370 \\
\hline Sedatives & $29(8.2)$ & $21(6.1)$ & $50(7.1)$ & 0.276 \\
\hline Hypnotics & $18(5.1)$ & $15(4.3)$ & $33(4.7)$ & 0.640 \\
\hline Mean prothrombin time $\%$ & $88.3 \pm 23.6$ & $90.5 \pm 22.0$ & $89.4 \pm 22.8$ & 0.211 \\
\hline INR & $1.2 \pm 0.7$ & $1.2 \pm 0.5$ & $1.2 \pm 0.6$ & 0.209 \\
\hline Mean platelet count $G \cdot L^{-1}$ & $318 \pm 149$ & $338 \pm 160$ & $329 \pm 156$ & 0.095 \\
\hline
\end{tabular}

Data are presented as mean \pm SD or $n(\%)$, unless otherwise stated. BMI: body mass index; ASA: American Society of Anesthesiologists; COPD: chronic obstructive pulmonary disease; LMWH: low molecular weight heparin; INR: international normalised ratio.

\section{Results}

Demographic data are presented in table 1. There were no significant differences between the two randomised groups in terms of age, sex, physical status, ASA class, presence of comorbidities or current medication. However, there was a trend towards a higher number of patients with haematological malignancy and hence immunosuppression in the bolus group. Of note, almost three-quarters of all patients were classified as ASA class III (bolus and infusion groups $74.9 \%$ and $72.3 \%$, respectively), defined as patients with severe systemic disease.

Table 2 shows the indication, number and distribution of diagnostic and interventional procedures per patient and randomisation group. The main reason for bronchoscopy was pulmonary infection, followed by suspicion of malignancy and interstitial lung disease. Accordingly, the most common diagnostic procedures 
TABLE 2 Main indications for bronchoscopy per randomisation group

\begin{tabular}{lcccc} 
Indication for bronchoscopy & Bolus & Infusion & Total & p-value \\
\hline Subjects $n$ & 355 & 347 & 702 & \\
Suspicion of malignancy & $83(23.4)$ & $93(26.8)$ & $176(25.1)$ & 0.296 \\
Interstitial lung disease & $29(8.2)$ & $48(13.8)$ & $77(10.7)$ & 0.016 \\
Infection & $130(36.6)$ & $107(30.8)$ & $237(33.8)$ & 0.105 \\
Chronic cough & $17(4.8)$ & $9(2.6)$ & $26(3.7)$ & 0.124 \\
Haemoptysis & $5(1.4)$ & $10(2.9)$ & $15(2.1)$ & 0.201 \\
Bronchial toilette & $35(9.9)$ & $35(10.1)$ & $70(10.0)$ & 0.920 \\
Stenting & $10(2.8)$ & $9(2.6)$ & $19(2.7)$ & 0.855 \\
Laser therapy & $5(1.4)$ & $3(0.9)$ & $8(1.1)$ & 0.725 \\
Miscellaneous & $41(11.5)$ & $32(9.2)$ & $73(10.4)$ & 0.312 \\
Diagnostic procedures & & & & \\
Inspection only & $23(6.5)$ & $23(6.6)$ & $46(6.6)$ & 1.000 \\
Bronchial washings & $99(28.0)$ & $83(23.9)$ & $182(26.0)$ & 0.222 \\
BAL & $214(60.3)$ & $212(61.3)$ & $426(60.8)$ & 0.788 \\
Bronchial brushing & $40(11.3)$ & $46(13.3)$ & $86(12.3)$ & 0.430 \\
Endobronchial biopsy & $48(13.5)$ & $56(16.1)$ & $104(14.8)$ & 0.329 \\
Transbronchial biopsy & $48(13.5)$ & $72(20.7)$ & $120(17.1)$ & 0.011 \\
Mediastinal TBNA & $24(6.8)$ & $36(10.4)$ & $60(8.5)$ & 0.087 \\
Peripheral TBNA & $15(4.2)$ & $20(5.8)$ & $35(5.0)$ & 0.349 \\
EBUS & $24(6.8)$ & $29(8.4)$ & $53(7.5)$ & 0.423 \\
Interventions & $6(1.7)$ & $7(2)$ & $13(1.9)$ & 0.748 \\
Laser therapy & $12(3.4)$ & $12(3.5)$ & $24(3.4)$ & 0.955 \\
Stenting & $2(0.6)$ & $2(0.6)$ & $4(0.6)$ & 0.982 \\
Calypso implantation & $3(0.8)$ & $4(1.2)$ & $7(1.0)$ & 0.682 \\
Valve implantation & $203(57.5)$ & $174(50.3)$ & $377(53.9)$ & \\
Number of procedures & $79(22.4)$ & $82(23.7)$ & $161(23.0)$ & 0.115 \\
1 & $48(13.6)$ & $67(19.4)$ & $115(16.5)$ & \\
2 & & & & \\
$\geqslant 3$ & & & & \\
\hline & & & & \\
\hline
\end{tabular}

Data are presented as $\mathrm{n}(\%)$, unless otherwise stated. BAL: bronchoalveolar lavage; TBNA: transbronchial needle aspiration; EBUS: endobronchial ultrasound.

were BAL $(60.8 \%)$ and bronchial washing $(26.0 \%)$, followed by transbronchial and endobronchial biopsies (17.1\% and $14.8 \%$, respectively). The majority of patients underwent one $(53.9 \%)$ or two $(23.0 \%)$ diagnostic bronchoscopic procedures. There were more diagnostic bronchoscopies for infection in the bolus group as compared with the infusion group, while more bronchoscopies for suspected interstitial lung disease were performed in the infusion group. Accordingly, the number of transbronchial biopsies was significantly higher in the infusion group (48 versus $72 ; \mathrm{p}=0.011$ ).

Incidence of adverse events and complications

The number (rate) of any adverse event was similar in both randomised groups $(219(61.7 \%)$ patients in the bolus group versus $211(60.8 \%)$ patients in the infusion group; $\mathrm{p}=0.810)$. There were complications in eight $(1.1 \%)$ cases (seven major bleedings (four in the bolus group and three in the infusion group), one respiratory failure (in the infusion group)) leading to the termination of examination with subsequent intubation and transfer to ICU in one patient in each group. Intubation was performed for major bleeding in one patient (bolus group) and for respiratory failure in another (infusion group). There were no deaths. Details of adverse events and complications are shown in table 3.

\section{Secondary end-points}

Medication requirements and duration of all interventions are shown in table 4. As compared with the bolus group, the amount of propofol required was significantly higher in the infusion group $(226 \pm 147 \mathrm{mg}$ versus $308 \pm 204.8 \mathrm{mg} ; \mathrm{p}<0.0001)$. In a linear multivariate regression model, this difference remained significant independently of duration and the interventions (e.g. transbronchial biopsy and endobronchial ultrasound) performed during flexible bronchoscopy. Both lidocaine and hydrocodone requirements were similar in both groups.

The duration of bronchoscopy (from beginning of sedation to removal of bronchoscope) was significantly longer in the infusion group (14 (9-24) min versus $17(12-27) \mathrm{min} ; \mathrm{p}<0.0001)$. This difference was mainly 


\begin{tabular}{|c|c|c|c|c|}
\hline & Bolus & Infusion & Total & p-value \\
\hline Subjects $n$ & 355 & 347 & 702 & \\
\hline \multicolumn{5}{|l|}{ Adverse events } \\
\hline Hypotension systolic pressure $\leqslant 90 \mathrm{mmHg}$ & $94(26.5)$ & $109(31.4)$ & $203(28.9)$ & 0.149 \\
\hline Hypoxaemia oxygen saturation $\leqslant 90 \%$ & $133(37.5)$ & 133 (38.3) & $266(37.9)$ & 0.814 \\
\hline $\begin{array}{l}\text { Insertion of nasopharyngeal/oropharyngeal } \\
\text { airway }\end{array}$ & $21(5.9)$ & $23(6.6)$ & $44(6.3)$ & 0.704 \\
\hline Pneumothorax & $0(0)$ & $0(0)$ & $0(0)$ & 1.000 \\
\hline Minor bleeding & $30(8.5)$ & $30(8.6)$ & $60(8.6)$ & 0.936 \\
\hline \multicolumn{5}{|l|}{ Complications } \\
\hline Major bleeding & $4(1.1)$ & $3(0.9)$ & $7(1.0)$ & 0.724 \\
\hline Termination of examination & $1(0.3)$ & $1(0.3)$ & $2(0.3)$ & 0.322 \\
\hline Intubation & $1(0.3)$ & $1(0.3)$ & $2(0.3)$ & 0.989 \\
\hline Transfer to intensive care unit & $1(0.3)$ & $1(0.3)$ & $2(0.3)$ & 0.322 \\
\hline Death & $0(0)$ & $0(0)$ & $0(0)$ & 1.000 \\
\hline
\end{tabular}

Data are presented as $\mathrm{n}(\%)$, unless otherwise stated.

due to a significantly longer phase between beginning of sedation and insertion of the bronchoscope, while no significant difference could be observed in terms of duration of the procedure itself (after insertion of the bronchoscope).

Figure 2 presents the haemodynamic findings before, during and after bronchoscopy. There was no significant difference in lowest oxygen saturation between the two groups, while lowest systolic and diastolic blood pressure were significantly lower in the infusion group (105 versus $100 \mathrm{mmHg}, \mathrm{p}=0.008$ and 60 versus $58 \mathrm{mmHg}, \mathrm{p}=0.014$, respectively). Of note, all oxygen saturation assessments, as well as systolic and diastolic blood pressure values, were significantly higher in the infusion group at the beginning of sedation.

The lowest respiratory rate was significantly higher in the bolus group (15 versus $13 ; \mathrm{p}=0.002$ ) as compared with the infusion group. There was no significant difference between the two groups in terms of lowest or highest heart rate.

Cough scores, as judged by the bronchoscopists, nursing staff and patients themselves, did not differ between patients randomised to the bolus and infusion group. Similarly, there were no differences in the perception of discomfort, anxiety and fitness related to the procedure, as well as readiness for repeating bronchoscopy across treatment groups. Interestingly, $96.3 \%$ of all patients would have agreed to undergo a further bronchoscopic examination (table 5).

Table 6 presents the reasons for episodic change in the study arm receiving propofol continuous infusion. Most changes (90\%) occurred due to perceived discomfort and persistent cough at 3 or 6 min $(57 \%)$ following start of sedation.

\section{TABLE 4 Bronchoscopy characteristics per randomisation group}

\begin{tabular}{|c|c|c|c|c|}
\hline Characteristics & Bolus & Infusion & Total & p-value \\
\hline Subjects $n$ & 355 & 347 & 702 & \\
\hline Propofol total mg & $226.6 \pm 147.0$ & $308.3 \pm 204.8$ & $267.0 \pm 182.5$ & $<0.0001$ \\
\hline Propofol $\mathrm{mg} \cdot \mathrm{kg}^{-1} \cdot \mathrm{min}^{-1}$ & $0.217 \pm 0.127$ & $0.235 \pm 0.137$ & $0.226 \pm 0.132$ & 0.069 \\
\hline Propofol as bolus $\mathrm{mg}$ & $226.6 \pm 147.0$ & $31.1 \pm 33.1$ & $128 \pm 143.7$ & $<0.0001$ \\
\hline Hydrocodone mg & $4.4 \pm 1.6$ & $4.6 \pm 1.6$ & $4.5 \pm 1.6$ & 0.058 \\
\hline $\begin{array}{l}\text { Duration from beginning of sedation to } \\
\text { removal of bronchoscope } \min \end{array}$ & $14(9-24)$ & $17(12-27)$ & $16(10-27)$ & $<0.0001$ \\
\hline $\begin{array}{l}\text { Duration from insertion to removal of } \\
\text { bronchoscope min }\end{array}$ & $11(7-22)$ & $13(7-24)$ & $12(7-22)$ & 0.062 \\
\hline
\end{tabular}

Data are presented as mean \pm SD or as median (interquartile range), unless otherwise stated. 

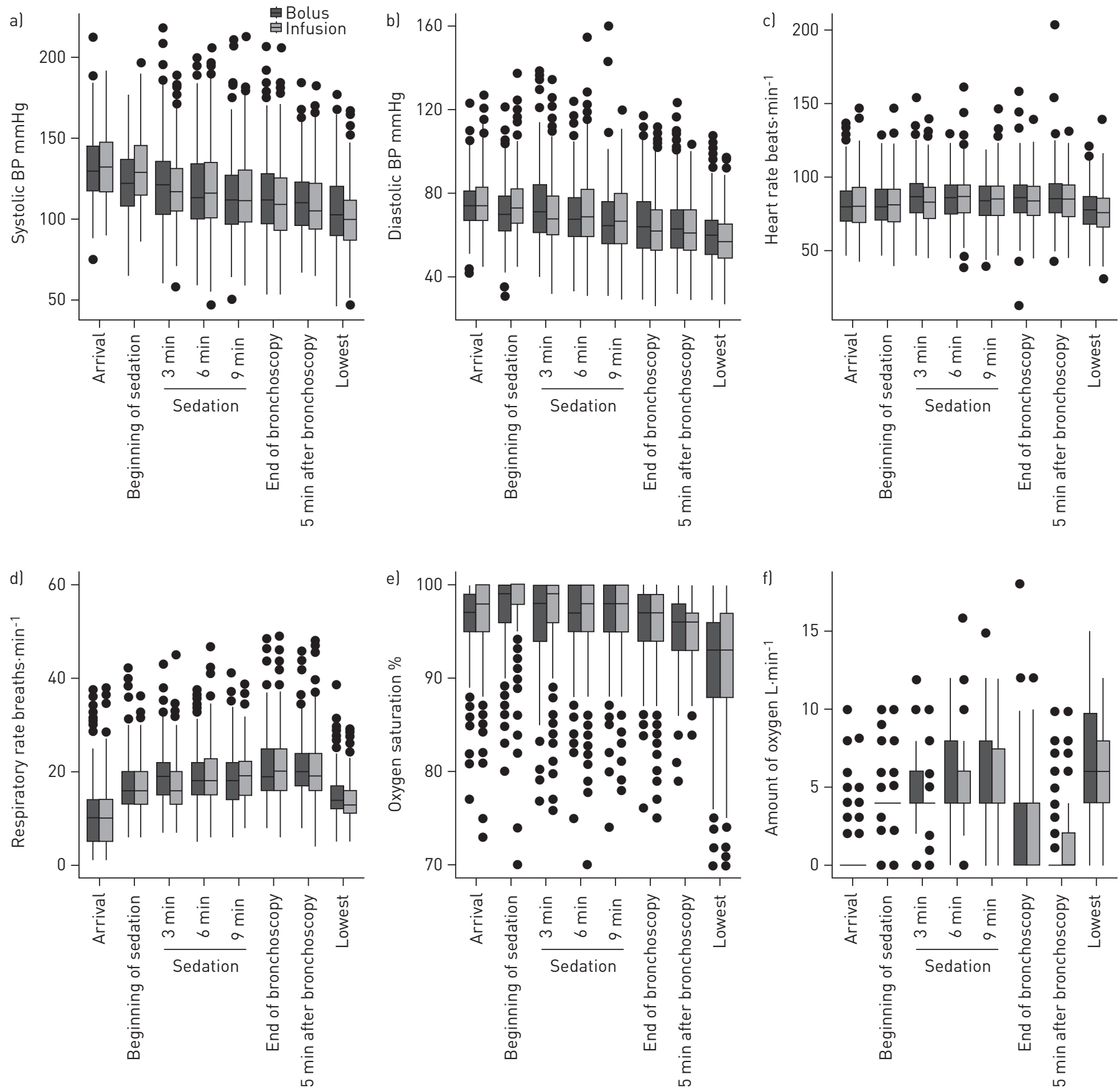

FIGURE 2 Haemodynamic parameters: a) systolic blood pressure (BP), b) diastolic BP, c) heart rate, d) respiratory rate, e) oxygen saturation and f) amount of oxygen per randomisation group.

\section{Discussion}

The present study demonstrates that the number of adverse events and complications is similar in patients receiving propofol using an intermittent bolus technique or continuous infusion for sedation in flexible bronchoscopy. However, patients receiving propofol as a continuous infusion required higher doses of propofol and presented a prolonged duration of bronchoscopy as compared with the intravenous bolus application group.

To our knowledge, this is the first randomised, controlled trial comparing bolus and continuous administration for conscious sedation with propofol in diagnostic and interventional bronchoscopy. Propofol has proved to be an attractive option to combined sedation with midazolam and hydrocodone, providing significantly faster recovery times and improved patient satisfaction scores $[11,18]$. It has been 
TABLE 5 Outcome parameters per randomisation group

\begin{tabular}{lcccc} 
Characteristics & Bolus & Infusion & Total & p-value \\
\hline $\begin{array}{l}\text { Subjects n } \\
\text { Cough score }\end{array}$ & 355 & 347 & 702 & \\
$\quad$ Physician VAS & $3.0(1.0-5.0)$ & $2.0(1.0-5.0)$ & $2.8(1.0-5.0)$ & 0.398 \\
$\quad$ Nurse VAS & $2.5(1.8-4.0)$ & $3.0(1.0-4.0)$ & $2.7(1.0-4.0)$ & 0.619 \\
$\quad$ Patient VAS & $3.0(1.0-6.0)$ & $3.0(1.0-6.0)$ & $3.0(1.0-6.0)$ & 0.917 \\
Discomfort score & $0.5(0-1.0)$ & $0.5(0-1.5)$ & $0.5(0-1.0)$ & 0.942 \\
Anxiety score & $0.5(0-2.0)$ & $0.5(0-1.5)$ & $0.5(0-2.0)$ & 0.737 \\
$\begin{array}{l}\text { Fitness score } \\
\text { Readiness for further bronchoscopic } \\
\quad \text { procedure }\end{array}$ & $3.75(2-6)$ & $3.5(2-5)$ & $3.5(2-5.5)$ & 0.644 \\
& $277(95.4)$ & $271(97.1)$ & $543(96.3)$ & 0.288
\end{tabular}

Data are presented as as median (interquartile range) or $\mathrm{n}(\%)$, unless otherwise stated. Data are presented for 562 patients. VAS: visual analogue scale.

also showed that the combination of propofol and hydrocodone is safe, has a better cough suppressing effect and is associated with significantly lower propofol requirements as compared with propofol alone [10]. The feasibility and safety of propofol sedation as administered by repeated bolus technique is also supported by two large cohort studies reporting the performance of propofol sedation in flexible bronchoscopy [14, 17].

In the current study, the number of adverse events observed during and following flexible bronchoscopy was similar for both sedation regimens, i.e. bolus and continuous infusion of propofol. Thereby, the most frequently observed adverse event was hypoxaemia, followed by hypotension and minor bleeding. This finding is in agreement with prior reports: we have previously described the incidence of hypoxaemia on at least one occasion during bronchoscopy to range between $29 \%$ and $32 \%$ in two large randomised studies $[10,11]$. Similar figures were reported by another smaller trial [15]. The frequency of hypoxaemia and hypotension as reported by CLARK et al. [18] were $34.9 \%$ and $4.7 \%$, respectively. Interestingly, a significantly lower incidence of hypoxaemia was reported by two cohort studies on propofol sedation in flexible bronchoscopy. Herein, BossLet et al. [14] found hypoxaemia in only $3.8 \%$ and hypotension in only $1 \%$ of all patients using a nurse administered propofol protocol [17]. Differences in the incidence of hypoxaemia and hypotension across the studies can be tentatively explained by several factors. First, in contrast to previous reports, we performed a large number of diagnostic and interventional procedures, which require stable yet well-sedated patients with minimal coughing. Moreover, almost one-third of our patients were severely immunocompromised, including several cases of HIV infection as well as active drug use. It is well known that patients with advanced oncological and haematological disease, including solid organ and bone marrow transplantation, intravenous drug users and HIV patients, have a higher incidence of complications during bronchoscopy [23]. Some patients with HIV infection require higher doses of sedation [6]. However, sedation requirements may depend on the anti-retrovirals being used and if they inhibit or augment the hepatic metabolism of propofol [24, 25]. Accordingly, the amount of propofol given in the current study $\left(0.217 \mathrm{mg} \cdot \mathrm{kg}^{-1} \cdot \mathrm{min}^{-1}\right.$ in the bolus group and $0.235 \mathrm{mg} \cdot \mathrm{kg}^{-1} \cdot \mathrm{min}^{-1}$ in the infusion group) was globally higher than the amount described in previous reports $\left(0.15 \mathrm{mg} \cdot \mathrm{kg}^{-1} \cdot \mathrm{min}^{-1}\right)[10,11,17,18]$ and particularly by BossLeT et al. [14]. Secondly, the distribution of patients within ASA classes may explain differences in the incidence of hypoxaemia and hypotension across the studies. Only roughly one-fifth of the patients in the current study were classified as ASA I or II, while the vast majority (84\%) of the patients included in the study by BossLet et al. [14] belonged to these two ASA classes, including normal healthy patients and

TABLE 6 Reasons for episodic change in infusion rate

\begin{tabular}{lccccc} 
Time & Hypotension & Hypoxaemia & Discomfort & Cough & Other \\
\hline Beginning of sedation & 0 & 3 & 26 & 9 & 4 \\
At 3 min & 0 & 12 & 71 & 45 & 6 \\
At $\mathbf{m i n}$ & 0 & 15 & 68 & 56 & 5 \\
At 9 min & 0 & 7 & 39 & 47 & 1 \\
At $>9$ min & 1 & 9 & 21 & 39 & 2 \\
\hline
\end{tabular}

Data are presented as $\mathrm{n}$. 
patients with mild systemic disease. Finally, the definition of "hypoxaemia" varied in the above mentioned studies $(<90 \%$ versus $\leqslant 90 \%)$. Moreover, the term hypoxaemia was used independent of its duration in the current paper, while other authors included duration of $>1$ or $2 \mathrm{~min}$ in the definition of hypoxaemia and desaturation, respectively. Although we have previously demonstrated that the incidence of adverse events observed with propofol is comparable with other sedation regimes, e.g. benzodiazepine and opiate or benzodiazepine alone [10,11], both hypoxaemia and hypotension are to be commonly expected in a considerable amount of severely ill patients undergoing bronchoscopy under propofol.

The incidence of bleeding in this study is in accordance with previous reports of ours but higher than reported by SCHLATTER et al. [10] and BOSSLET et al. [14], probably reflecting the widespread use of transbronchial and endobronchial forceps biopsies in our institution. Indeed, from the severe complications noted in eight patients (1.1\%, four in each group), seven were major bleedings. It is noteworthy that the vast majority of complications were "intervention" related and not sedation related. Overall, the number of complications observed within this trial is consistent with previous results reported in the literature $[10,11,17]$.

The difference of duration of $3 \mathrm{~min}$, although statistically significant, is small. However, when considering that, in several centres, many procedures are performed each day, we leave it up to the reader to judge whether this difference is or is not significant for their setting.

The amount of propofol required for sedation was significantly higher in the infusion group (226 $\pm 147 \mathrm{mg}$ versus $308 \pm 204.8 \mathrm{mg}, \mathrm{p}<0.0001)$. In a linear multivariate regression model, this difference remained significant, independently of duration and the interventions performed during flexible bronchoscopy. One could argue that the applied regimen itself lead to a higher dose of propofol in the infusion group. However, sedation in the infusion group was much more often considered insufficient than too deep (table 6), leading to an increase or failure to decrease the infusion rate or the application of additional propofol boluses. The most plausible explanation for this observation is a failure to reach a blood concentration of propofol of $>1 \mu \mathrm{g} \cdot \mathrm{mL}^{-1}$, usually leading to sedation, if propofol is given as an infusion as compared with bolus application. Moreover, the initial bolus of $10 \mathrm{mg}$ of propofol given to those patients was low. Application of higher boluses usually leads to a rapid rise in plasma concentration above this critical level. Therefore, it cannot be excluded that this initial low dose bolus has led to higher doses than expected being used and to more adverse events in the continuous infusion rate arm. It is also a reason why it may have taken longer for these patients to achieve adequate sedation. A change in protocol could therefore have led to a different result and conclusion regarding the duration of the sedation.

Hydrocodone $4 \mathrm{mg}$ was routinely given to all patients. We have previously shown in two randomised studies that hydrocodone, both in combination with midazolam and propofol, markedly reduced cough during flexible bronchoscopy without causing significant desaturation $[8,10]$. Hydrocodone was used as it is much cheaper than the newer opiates.

The present study has a few limitations. There was an imbalance in the distribution of indication for bronchoscopy with more diagnostic bronchoscopies being performed for infection in the bolus group as compared with the infusion group, while more bronchoscopies were performed for suspected interstitial lung disease in the infusion group. Accordingly, the number of transbronchial biopsies was significantly higher in the infusion group ( 48 versus $72 ; \mathrm{p}=0.011$ ). However, assuming a higher rate of complications in patients undergoing transbronchial biopsy as compared with patients undergoing BAL only, the incidence of adverse events and complications in the infusion group was overestimated rather than underestimated. Another factor to consider is that this was a monocentric study performed in an institution in which the nursing staff has considerable expertise with propofol sedation for endoscopic procedures. Hence, caution might be needed when introducing this sedative regimen in other institutions with less experienced nursing staff. No sedation score was used to assess the level of sedation achieved, thereby following the recommendations of the ASA [26], which suggest that no specific score other than the response of the patient to verbal commands or tactile stimulation is required for monitoring sedation. As suggested by the same society, we have provided the recommended monitoring of patients with pulseoxymetry, blood pressure measurements at defined intervals as wells as electrocardiographic monitoring. Of note, electroencephalogram-guided propofol administration for flexible bronchoscopy (where the goal was to achieve and maintain a bispectral index between 70 and 85 ) was shown to be safe in a study by CLARK et al. [18]. Moreover, this study was nonblinded and this may be a source of bias. Although blinding of the study medication might have enhanced the robustness of our findings, it represented an insurmountable obstacle for the study realisation. There are no data about the distribution of the procedures among pulmonary fellows and attending physicians. Similarly, data about the distribution of the nurses administering the sedation in each trial arm is not available. Finally, neither the nurse nor the physician was blinded to the type of sedation received so that their VAS ratings of cough are limited. However, due to randomisation and the fact that, if any, this bias can be considered a non-differential misclassification bias, i.e. meaning that the 
error rate or probability of being misclassified is probably the same for all study subjects, it would have produced a conservative bias. In the case of binary or dichotomous variables, this would probably result in an underestimate of the hypothesised relationship between exposure and outcome. Finally, the severity of chronic obstructive pulmonary disease was not systematically recorded within the study records and capnography was not performed in the present study. Therefore, no statement can be made about the risk of type II respiratory failure.

The strengths of the present study are the large number of patients with mixed, relevant comorbidities; the diversity of diagnostic and interventional bronchoscopic procedures, the completeness of the evaluation with no lost to follow-up and the original randomised non-inferiority design. Finally, well defined, "hard" end-points, such as need of ICU, intubation or death were chosen, which appears even more important in the absence of blinding of the study.

In conclusion, our data suggest that propofol given as a continuous infusion for conscious sedation in flexible bronchoscopy is as safe as bolus administration. However, with the current reported regimen, it is associated with higher propofol requirements and a longer duration of the bronchoscopy.

\section{References}

1 Honeybourne D, Babb J, Bowie P, et al. British Thoracic Society guidelines on diagnostic flexible bronchoscopy. Thorax 2001; 56: I1-I21.

2 Gonzalez R, De-La-Rosa-Ramirez I, Maldonado-Hernandez A, et al. Should patients undergoing a bronchoscopy be sedated? Acta Anaesth Scand 2003; 47: 411-415.

3 Putinati S, Ballerin L, Corbetta L, et al. Patient satisfaction with conscious sedation for bronchoscopy. Chest 1999; 115: $1437-1440$.

Matot I, Kramer MR. Sedation in outpatient bronchoscopy. Resp Med 2000; 94: 1145-1153.

Pickles J, Jeffrey M, Datta A, et al. Is preparation for bronchoscopy optimal? Eur Respir J 2003; 22: 203-206.

6 Chhajed PN, Wallner J, Stolz D, et al. Sedative drug requirements during flexible bronchoscopy. Respiration 2005; 72: 617-621.

7 Stolz D, Chhajed N, Leuppi J, et al. Nebulized lidocaine for flexible bronchoscopy - a randomized, double-blind, placebo-controlled trial. Chest 2005; 128: 1756-1760.

8 Stolz D, Chhajed PN, Leuppi JD, et al. Cough suppression during flexible bronchoscopy using combined sedation with midazolam and hydrocodone: a randomised, double blind, placebo controlled trial. Thorax 2004; 59: 773-776.

9 Greig JH, Cooper SM, Kasimbazi HJN, et al. Sedation for fiber optic bronchoscopy. Respir Med 1995; 89: 53-56.

10 Schlatter L, Pflimlin E, Fehrke B, et al. Propofol versus propofol plus hydrocodone for flexible bronchoscopy: a randomised study. Eur Respir J 2011; 38: 529-537.

11 Stolz D, Kurer G, Meyer A, et al. Propofol versus combined sedation in flexible bronchoscopy: a randomised noninferiority trial. Eur Respir J 2009; 34: 1024-1030.

12 Fulton B, Sorkin EM. Propofol - an overview of its pharmacology and a review of its clinical efficacy in intensivecare sedation. Drugs 1995; 50: 636-657.

13 Shelley MP, Wilson P, Norman J. Sedation for fiberoptic bronchoscopy. Thorax 1989; 44: 769-775.

14 Bosslet GT, DeVito ML, Lahm T, et al. Nurse-administered propofol sedation: feasibility and safety in bronchoscopy. Respiration 2010; 79: 315-321.

15 Clarkson K, Power CK, Oconnell F, et al. A comparative evaluation of propofol and midazolam as sedative agents in fiberoptic bronchoscopy. Chest 1993; 104: 1029-1031.

16 Bryson HM, Fulton BR, Faulds D. Propofol - an update of its use in anesthesia and conscious sedation. Drugs 1995; 50: 513-559.

17 Grendelmeier P, Kurer G, Pflimlin E, et al. Feasibility and safety of propofol sedation in flexible bronchoscopy. Swiss Med Wkly 2011; 141: w13248.

18 Clark G, Licker M, Younossian AB, et al. Titrated sedation with propofol or midazolam for flexible bronchoscopy: a randomised trial. Eur Respir J 2009; 34: 1277-1283.

19 Kiriyama S, Gotoda T, Sano H, et al. Safe and effective sedation in endoscopic submucosal dissection for early gastric cancer: a randomized comparison between propofol continuous infusion and intermittent midazolam injection. J Gastroenterol 2010; 45: 831-837.

20 Kongkam P, Rerknimitr R, Punyathavorn S, et al. Propofol infusion versus intermittent meperidine and midazolam injection for conscious sedation in ERCP. J Gastrointest Liver 2008; 17: 291-297.

21 Chhajed PN, Glanville AR. Management of hypoxemia during flexible bronchoscopy. Clin Chest Med 2003; 24: 511-516.

22 Heuss LT, Schnieper P, Drewe J, et al. Safety of propofol for conscious sedation during endoscopic procedures in high-risk patients-a prospective, controlled study. Am J Gastroenterol 2003; 98: 1751-1757.

23 White P, Bonacum JT, Miller CB. Utility of fiberoptic bronchoscopy in bone marrow transplant patients. Bone Marrow Transplant 1997; 20: 681-687.

24 Jose RJ, Marshall N, Lipman MC. Important antiretroviral drug interactions with benzodiazepines used for sedation during bronchoscopy. Chest 2012; 141: 1125.

25 Hsu AJ, Carson KA, Yung R, et al. Severe prolonged sedation associated with coadministration of protease inhibitors and intravenous midazolam during bronchoscopy. Pharmacotherapy 2012; 32: 538-545.

26 American Society of Anesthesiologists Task Force on Sedation and Analgesia by Non-Anesthesiologists. Practice guidelines for sedation and analgesia by non-anesthesiologists. Anesthesiology 2002; 96: 1004-1017. 